\title{
The usefulness of selected biomarkers in patients with valve disease
}

\author{
Piotr Duchnowski*,1, Tomasz Hryniewiecki ${ }^{1}$, Mariusz Kuśmierczyk ${ }^{2}$ \& Piotr Szymański ${ }^{1}$ \\ ${ }^{1}$ Department of Acquired Cardiac Defects, Institute of Cardiology, Warsaw, Poland \\ ${ }^{2}$ Department of Cardiosurgery \& Transplantology, Institute of Cardiology, Warsaw, Poland \\ *Author for correspondence: duchnowski@vp.pl
}

\begin{abstract}
Aim: The aim of the study was to investigate the prognostic value of selected biomarkers in patients undergoing valve surgery. Materials \& methods: A prospective study was conducted on a group of consecutive patients with hemodynamically significant valve defects that underwent elective valve repair or replacement surgery. The primary end point was any major adverse event including death within 30 days. Results: The study group included 416 patients. The composite end point occurred in 81 patients. At multivariate analysis high-sensitivity $C$-reactive protein $(p=0.03)$, red cell distribution width $(p=0.0001)$ and red blood cell count $(p=0.005)$ remained independent predictors of the primary end point. Conclusion: Elevated high-sensitivity C-reactive protein, red cell distribution width and red blood cell count were associated with a poorer outcome following valve surgery.
\end{abstract}

First draft submitted: 27 March 2018; Accepted for publication: 5 July 2018; Published online: 6 December 2018

Keywords: biomarkers $\bullet$ EuroSCORE $\| \bullet$ high-sensitivity C-reactive protein $\bullet$ postoperative complications $\bullet$ predictors - red blood cell count $\bullet$ red cell distribution width $\bullet$ risk stratification $\bullet$ valve disease $\bullet$ valve surgery

In the risk assessment of cardiac surgery, in European conditions, EuroSCORE II is most often used [1]. The reliability of these calculators in patients qualified for cardiac valvular surgery is limited. There are significant discrepancies between the risk of surgery predicted using risk calculators and actual mortality [2-7]. There is therefore a need to look for more accurate tools to determine risk in patients who are eligible for valvular surgery. In clinical practice in various areas of cardiology, the risk assessment strategy using biological markers is of great interest. The usefulness of selected biological markers in patients with heart valve disease in the stratification of operational risk has been described in numerous studies [8-11].

High-sensitivity C-reactive protein (hsCRP) is a one of the most important acute phase protein which adds prognostic information on cardiovascular risk. Studies have demonstrated a relationship between higher values of hsCRP and worse prognosis in patients with coronary artery disease, myocardial infarction, heart failure, atrial fibrillation, arterial hypertension, pulmonary embolism or diabetes $[12,13]$. On the other hand, the prognostic significance of preoperative hs-CRP levels in patients with severe symptomatic valve disease undergoing valve surgery in a 30-day follow-up is not established. Because there is a need to supplement the risk assessment tools in patients with valve disease qualified for surgery, we tried to check the suitability of selected biomarkers in this group of patients.

\section{Materials \& methods}

This was a prospective study of consecutive patients with hemodynamically significant valve disease (aortic stenosis, aortic regurgitation, mitral stenosis and mitral regurgitation) and without significant atherosclerotic changes in the coronary arteries that subsequently underwent elective replacement or repair of the valve. The exclusion criteria were: a lack of consent to participate in the study, significant atherosclerotic changes in the coronary arteries identified by angiography, patients under 18 years of age, autoimmune diseases, chronic inflammatory bowel, active neoplastic diseases and active endocarditis. The risk of surgery using EuroSCORE II was calculated for each patient. The day before surgery a blood sample for biomarkers was collected from each patient. Complete blood count was performed with K2-EDTA samples, using a Cobas 6000 electronic counter (Roche, Mannheim, Germany). 
The plasma levels of C-reactive protein (CRP) concentrations were measured by the Cardiac C-Reactive Protein (Latex) High Sensitive Test (Roche). All procedures were performed through a midline sternotomy incision under general anesthesia in a normothermia. The primary composite end point consisted of death from all causes as well as: perioperative myocardial infarction (defined as the development of new $Q$ waves in two or more leads on an ECG, or alterations of myocardial contractility that did not previously exist in echocardiography), hemodynamic instability (defined as the need for a supply of mechanical circulatory support - intra-aortic balloon pump or extracorporeal membrane oxygenation), stroke (evidence of a new neurological deficit or a transient ischemic attack, confirmed by an imaging test), perioperative renal failure (requiring renal replacement therapy), prolonged mechanical ventilation (either mechanical ventilatory support lasting longer than $24 \mathrm{~h}$, or the need for reintubation) and the occurrence of multiple-organ failure (the dysfunction of two or more organs - based on laboratory parameters and/or the need to use organ replacement therapy). Patients were followed by direct observation during hospitalization, telephone interviews or clinic visits for 30 days after the surgery. The study was conducted at the Institute of Cardiology, Warsaw. The protocol was approved by The Institutional Ethics Committee.

\section{Statistical analysis}

A statistical analysis was performed using SAS version 9.2. Data are presented as the mean \pm standard deviation and the frequency (\%). Intergroup comparisons were made using the Mann-Whitney U test, the Pearson's $\chi^{2}$ test or Student's t-test. Logistic regression was used to assess relationships between variables. The following preoperative covariates: age, bilirubin, left ventricular ejection fraction, New York Heart Association classes, creatinine, hs-CRP, high-sensitivity troponin $\mathrm{T}$ (hs-TnT), hematocrit, hemoglobin, mean corpuscular hemoglobin, mean corpuscular hemoglobin concentration, mean corpuscular volume, $\mathrm{N}$-terminal of the prohormone brain natriuretic peptide, platelets, red cell distribution width (RDW), red blood cell count (RBC) and white blood cell count were investigated for association with the endpoints in univariate analysis. Significant determinants $(\mathrm{p}<0.05)$ identified from univariate analysis were subsequently entered into multivariate models. receiver-operating characteristic (ROC) curves were plotted for the EuroSCORE II alone and for the model combined of EuroSCORE II, hs-CRP, RDW and RBC for 30-day survival without major complications, including death following valve surgery. The additional predictive values of hs-CRP, RDW and RBC were assessed by a comparison of the areas under the ROC of the respective curves.

\section{Results}

The study included 416 patients who underwent valve surgery without concomitant procedures on coronary arteries. The mean age in the study group was $62.4( \pm 12.1) .16(3.8 \%)$ of the patients in the study had a previous myocardial infarction, but currently none of the patients had significant atherosclerotic changes in the coronary arteries. The mean plasma preoperative hs-CRP level was $0.38 \mathrm{mg} / \mathrm{dl}$ (standard deviation \pm 0.29 ). Baseline characteristics of the patients are presented in Table 1. The primary end point occurred in 81 patients: perioperative renal failure in 23 patients, prolonged mechanical ventilation in 44 patients, stroke in seven patients and mechanical circulatory support in 13 patients. Multi-organ failure was observed in 35 patients. Myocardial infarction occurred in seven patients. 12 patients died during the follow-up period as a result of gradually increasing multi-organ failure. More than one major complication occurred in 26 patients. The actual mortality was $2.8 \%$ versus the $3.3 \%$ mortality predicted by the EuroSCORE II model. Statistically significant predictors of combined end point at univariate analysis are presented in Table 2. At multivariate analysis hs-CRP (odds ratio [OR]: 2.554; 95\% CI: 1,454-3.654; $\mathrm{p}=0.03$ ), RDW (OR: 3.232; 95\% CI: 2.466-3.998; $\mathrm{p}=0.0001$ ) and RBC (OR: 0.398; 95\% CI: $0.216-0.578 ; \mathrm{p}=0.005)$ remained independent predictors of the composite end point. A positive correlation was found between the level of hs-CRP and RDW $(r=0.21 ; \mathrm{p}=0.009)$ as well as between the level of hs-CRP and hs-TnT $(\mathrm{r}=0.23 ; \mathrm{p}<0.0001)$, creatinine $(\mathrm{r}=0.25 ; \mathrm{p}=0.01)$ and NTproBNP $(\mathrm{r}=0.3 ; \mathrm{p}<0.0001)$. Hs-CRP, RDW, RBC and combined with EuroSCORE II was a better predictor of 30-day major complications (area under receiver operator characteristic [AUC-ROC] curve: 0.776; 95\% CI: 0.728-0.811) compared with EuroSCORE II alone (AUC-ROC curve: 0.741; 95\% CI: 0.695-0.792).

Figure 1 shows the areas under receiver operator characteristic curves of EuroSCORE II and the combined model $\mathrm{CRP}+\mathrm{RDW}+\mathrm{RBC}+$ EuroSCORE II for 30-day major complications including death. 


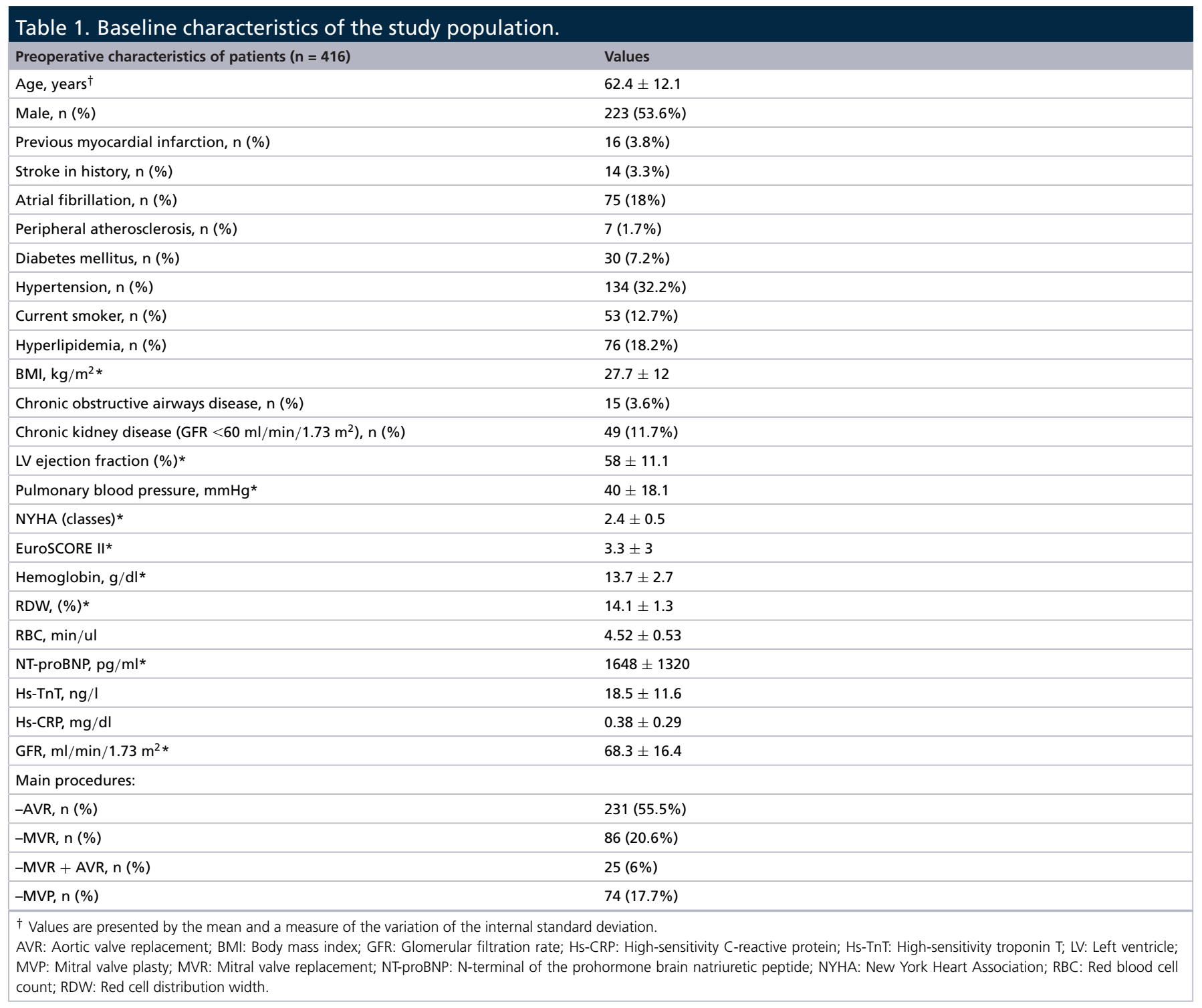

\section{Table 2. Univariate analysis of predictive factors for the occurrence of the composite end point.} Variable Odds ratio $95 \% \mathrm{Cl}$

Bilirubin, $(\mathrm{mg} / \mathrm{dl})$

$1.026-1.082$ p-value

Hgb, (g/dl) 1.054

0.600-0.802

0.0001

EF, (\%)

0.694

0.600-0.802

0.0001

Hs-CRP, (mg/dl)

0.965

0.948-0.983

0.0001

RBC, (mln/ul)

2.654

1.546-4.556

0.0001

RDW, (\%)

3.506

0.260-0.624

0.0001

EF: Ejection fraction; Hgb: Hemoglobin; Hs-CRP: High-sensitivity C-reactive protein; RBC: Red blood cell count, RDW: Red cell distribution width.

\section{Discussion}

The risk calculators are used to assess the surgical risk of heart valve surgery, in European conditions, these are EuroSCORE and EuroSCORE II, less often American Society of Thoracic Surgeons (STS). However, available literature indicates significant limitations on both the EuroSCORE scale and the improved version - EuroSCORE II and the American STS [1-7]. This prospective work, carried out on a group of 416 patients with valvular heart disease, without significant changes in the coronary arteries, subjected to heart valve surgery is an assessment of the 


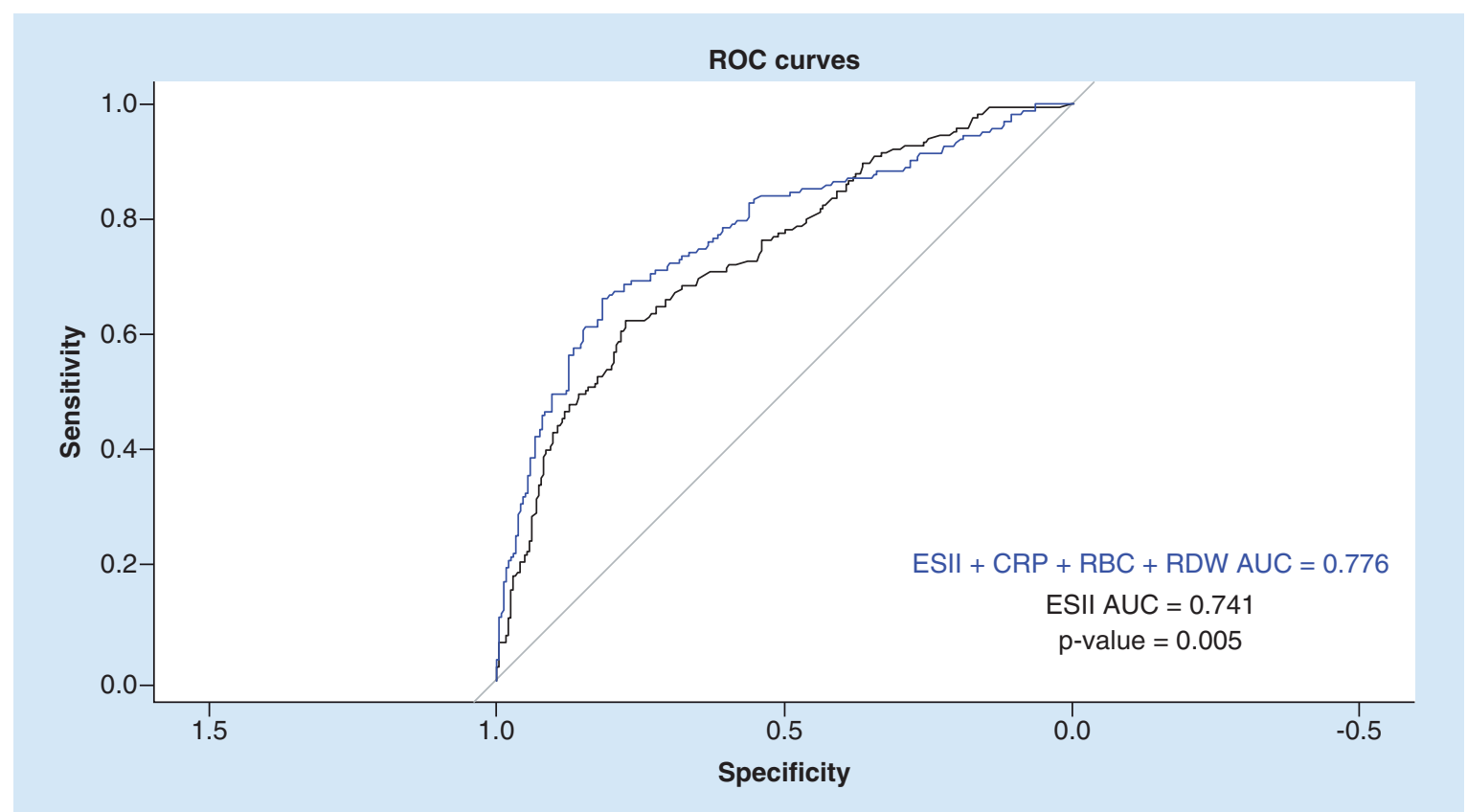

Figure 1. Areas under receiver-operatoring characteristic curves of EuroSCORE II and combined model EuroSCORE II + CRP + RDW + RBC for 30-day major complications following valve surgery.

AUC: Area under curve; ESII: EuroSCORE II; RBC: Red blood cell count; RDW: Red cell distribution width; ROC: Receiver-operating characteristic curve.

For color figures please see online at: https://www.futuremedicine.com/doi/10.2217/bmm-2018-0101

usefulness of selected biomarkers and blood morphology parameters in predicting death and serious postoperative complications in the 30-day observation.

In the presented study, the parameter hsCRP was found to be an independent predictor of death and serious postoperative complications. CRP is a cyclic pentamer, belonging to the family of pentaxins - ligand-binding proteins in calcium-dependent reactions [14]. Synthesis of CRP takes place primarily in the liver in response to pro-inflammatory factors IL-1 and IL-6. CRP is one of the most important acute phase proteins whose role is to participate in the body's immune response by facilitating the binding of complement $[15,16]$. In the available literature, among others, that elevated values of CRP and IL- 6 are predictors of inferior physical fitness and cognitive functions $[17,18]$. Numerous studies have also documented that chronic inflammation is associated with increased morbidity and mortality $[19,20]$. It has been described so far that factors, such as diabetes, sedentary lifestyle, smoking or obesity are associated with elevated CRP values [21-24]. However, not all patients with elevated CRP values are characterized by increased morbidity. The available literature suggests that morbidity in patients with elevated inflammation parameters results from an imbalance between proinflammatory and anti-inflammatory factors $[25,26]$. An important influence on chronic inflammation may be played by modifying factors, such as diet, level of physical activity, fat content in the body, smoking or genetic background $[27,28]$. The available literature shows that the lowest levels of CRP are found in people with normal body mass, and the relationship between BMI and proinflammatory factors takes the form of the letter U. People with the lowest morbidity and mortality are characterized by normal weight or overweight. In turn, persons with extreme weight groups are at least partially characterized by systemic inflammation $[29,30]$.

In previous studies, systemic inflammatory response syndrome predicts after transcatheter aortic valve implantation (TAVI) has been shown to increase mortality in short-term observation. In addition, it has been shown that elevated CRP values measured after aortic valve replacement are associated with worse prognosis [31,32]. So far, the usefulness of the preoperative level of the hs-CRP parameter in patients undergoing heart valve surgery has not been described unambiguously. The results of the presented study indicate the usefulness of biomarker determination, such as hs-CRP, RDW and RBC. These parameters turned out to be independent predictors of serious postoperative complications, including death in a 30-day observation. What is also important, it has been shown that adding these parameters to the EuroSCORE II calculation result increases the predictive power of this 
commonly used calculator, for predicting serious postoperative complications, including death. The study showed a significant correlation between the hs-CRP inflammatory parameter and parameters, such as RDW, hs-TnT, creatinine and $\mathrm{N}$-terminal of the prohormone brain natriuretic peptide. These results suggest that in patients with valvular heart disease, a chronic inflammatory process may have a negative impact on the hematopoietic system, cardiovascular system and renal function, followed by the occurrence of early postoperative complications. It is worth noting that the cause of all deaths was the increasing multi-organ failure, which occurred in 35 patients during the observation period.

\section{Summary points}

- This is a single-center study with a limited number of patients.

- High-sensitivity C-reactive protein (hSCRP) is a one of the most important acute phase proteins, which adds prognostic information on cardiovascular risk.

- The prognostic significance of preoperative hs-CRP levels in patients with severe symptomatic valve disease undergoing valve surgery in a 30-day follow-up is not established.

- The results of this study indicate that the hs-CRP, red cell distribution width and red blood cell count are useful parameters for estimating the risk of postoperative complications including death in patients with valvular heart disease.

- The predictive abilities of hs-CRP, red blood cell count and red cell distribution width assessed by the area under the receiver-operating characteristic curve, enhance the predictive ability of the EuroSCORE II calculator.

- In future studies, enlarging the group may allow to confirm the obtained results and develop a risk calculator using the parameters of inflammation.

- Further studies are needed regarding the usefulness of inflammation parameters as predictors of complications in patients with heart valve disease undergoing cardiac surgery.

- The results of our research may be helpful in the perioperative strategy in patients with valvular heart disease.

Financial \& competing interests disclosure

The authors have no relevant affiliations or financial involvement with any organization or entity with a financial interest in or financial conflict with the subject matter or materials discussed in the manuscript. This includes employment, consultancies, honoraria, stock ownership or options, expert testimony, grants or patents received or pending, or royalties.

No writing assistance was utilized in the production of this manuscript.

\section{Ethical conduct}

The authors state that they have obtained appropriate institutional review board approval or have followed the principles outlined in the Declaration of Helsinki for all human or animal experimental investigations. In addition, for investigations involving human subjects, informed consent has been obtained from the participants involved.

\section{References}

Papers of special note have been highlighted as: $\bullet$ of interest; $\bullet \bullet$ of considerable interest

1 Nashef SA, Roques F, Sharples LD et al. EuroSCORE II. Eur. J. Cardiothorac. Surg. 41(4), 734-44 (2012).

2 Van Gameren M, Kappetein A, Steyerberg E, Venema A, Berenschot E, Hannan E. Do we need separate risk stratification models for hospital mortality after heart valve surgery? Ann. Thorac. Surg. 85(3), 921-930 (2008).

3 Kuwaki K, Inaba H, Yamamoto T, Matsumura T, Morita T, Amano A. Performance of the EuroSCORE II and the Society of Thoracic Surgeons Score in patients undergoing aortic valve replacement for aortic stenosis. J. Cardiovasc. Surg. (Torino) 56(3), 455-462 (2015).

4 Borracci RA, Ingino C, Allende N, Ahuad Guerrero R. Prospective validation of EuroSCORE II in patients undergoing cardiac surgery in Argentinean centres. Interact. Cardiovasc. Thorac. Surg. 18(5), 539-543 (2014).

5 Grant S, Hickey G, Dimarakis I et al. Performance of the EuroSCORE models in emergency cardiac surgery. Circ. Cardiovasc. Qual. Outcomes 6(2), 178-185 (2013).

6 Chalmers J, Pullan M, Fabri B et al. Validation of EuroSCORE II in a modern cohort of patients undergoing cardiac surgery. Eur. J. Cardiothorac. Surg. 43(4), 688-694 (2013).

7 Parolari A, Pesce L, Trezzi M et al. EuroSCORE performance in valve surgery: a meta-analysis. Ann. Thorac. Surg. 89(3), 787-793 (2010).

8 Duchnowski P, Hryniewiecki T, Kuśmierczyk M, Szymański P. Red cell distribution width is a prognostic marker of perioperative stroke in patients undergoing cardiac valve surgery. Interact. Cardiovasc. Thorac. Surg. 25(6), 925-929 (2017). 
-. The results of this study indicate that the red cell distribution width is a useful parameter for estimating the risk of perioperative stroke and stroke-related mortality in patients with valvular heart disease.

9 Petaja L, Rosio H, Mildh L et al. Predictive value of high-sensitivity troponin T in addition to EuroSCORE II in cardiac surgery. Interact. Cardiovasc. Thorac. Surg. 23(1), 133-141 (2016).

10 Perreas K, Samanidis G, Dimitriou S et al. NT-proBNP in the mitral valve surgery. Crit. Pathw. Cardiol. 13(2), 55-61 (2014).

11 Duchnowski P, Hryniewiecki T, Zatorska T, Zebrowska A, Kuśmierczyk M, Szymanski P. High-sensitivity troponin T as a prognostic marker in patients undergoing aortic valve replacement. Pol. Arch. Intern. Med. 127(9), 628-630 (2017).

- The results of this study demonstrate that the addition of the preoperative high-sensitivity troponin $T$ measurement to EuroSCORE II may improve the ability of predicting mortality in patients with aortic stenosis who were treated surgically.

12 Ridker PM. A test in context: high-sensitivity C-reactive protein. J. Am. Coll. Cardiol. 67(6), 712-723 (2016).

13 Adukauskiene D, Čiginskiene A, Adukauskaite A, Pentiokiniene D, Šlapikas R, Čeponiene I. Clinical relevance of high sensitivity C-reactive protein in cardiology. Medicina 52(1), 1-10 (2016)

14 Osmand A, Friedenson B, Gewurz H, Painter R, Hofmann T, Shelton E. Characterization of C-reactive protein and the complement subcomponent $\mathrm{C} 1 \mathrm{t}$ as homologous proteins displaying cyclic pentameric symmetry (pentraxins). Proc. Natl Acad. Sci. USA 74(2), 739-743 (1977).

15 Volanakis JE. Human C-reactive protein: expression, structure, and function. Mol. Immunol. 38(2-3), 189-197 (2001)

- $\quad \mathrm{C}$-reactive protein is one of the most important acute phase proteins.

16 Boncler M, Luzak B, Watała C. Role of C-reactive protein in atherogenesis. Postepy Hig. Med. Dosw. 60, 538-546 (2006).

17 Puzianowska-Kuznicka M, Owczarz M, Wieczorowska-Tobis K et al. Interleukin-6 and C-reactive protein, successful aging, and mortality: the PolSenior study. Immun. Ageing 13, 21 (2016).

18 Velissaris D, Pantzaris N, Koniari I et al. C-Reactive protein and frailty in the elderly: a literature review. J. Clin. Med. Res. 9(6), 461-465 (2017).

-. Elevated values of C-reactive protein is a predictor of inferior physical fitness and cognitive functions.

19 Bruunsgaard $\mathrm{H}$. The clinical impact of systemic low-level inflammation in elderly populations. With special reference to cardiovascular disease, dementia and mortality. Dan. Med. Bull. 53(3), 285-309 (2006).

20 Beyer I, Njemini R, Bautmans I, Demanet C, Bergmann P, Mets T. Inflammation-related muscle weakness and fatigue in geriatric patients. Exp. Gerontol. 47(1), 52-59 (2012).

- Chronic inflammation is associated with increased morbidity and mortality.

21 Esposito K, Pontillo A, Di Palo C et al. Effect of weight loss and lifestyle changes on vascular inflammatory markers in obese women: a randomized trial. JAMA 289(14), 1799-1804 (2003).

22 Haffner S, Greenberg A, Weston W, Chen H, Williams K, Freed M. Effect of rosiglitazone treatment on nontraditional markers of cardiovascular disease in patients with Type 2 diabetes mellitus. Circulation 106(6), 679-684 (2002).

23 Rohde L, Hennekens C, Ridker P. Survey of C-reactive protein and cardiovascular risk factors in apparently healthy men. Am. J. Cardiol. 84(9), 1018-1022 (1999).

24 Tchernof A, Nolan A, Sites C, Ades P, Poehlman E. Weightloss reduces C-reactive protein levels in obese postmenopausal women. Circulation 105(5), 564-569 (2002).

25 Salvioli S, Monti D, Lanzarini C et al. Immune system, cell senescence, aging and longevity - inflammaging reappraised. Curr. Pharm. Des. 19(9), 1675-1679 (2013).

26 Minciullo P, Catalano A, Mandraffino G et al. Inflammaging and anti-inflammaging: the role of cytokines in extreme longevity. Arch. Immunol. Ther. Exp. 64(2), 111-126 (2016).

27 Baylis D, Bartlett DB, Patel HP, Roberts HC. Understanding how we age: insights into inflammaging. Longev. Healthspan 2(1), 8 (2013).

28 Dato S, Bellizzi D, Rose G, Passarino G. The impact of nutrients on the aging rate: a complex interaction of demographic, environmental and genetic factors. Mech. Ageing Dev. 154, 49-61 (2016).

29 Akin I, Nienaber CA. Obesity paradox in coronary artery disease. World J. Cardol. 7(10), 603-608 (2015).

30 Costanzo P, Cleland JG, Pellicori P et al. The obesity paradox in Type 2 diabetes mellitus: relationship of body mass index to prognosis: a cohort study. Ann. Intern. Med. 162(9), 610-618 (2015).

31 Rettig $\mathrm{T}$, Rigter $\mathrm{S}$, Nijenhuis $\mathrm{V}$ et al. The systemic inflammatory response syndrome predicts short-term outcome after transapical transcatheter aortic valve implantation. J. Cardiothorac. Vasc. Anesth. 29(2), 283-287 (2015).

-. Elevated values of $\mathrm{C}$-reactive protein measured after aortic valve replacement are associated with worse prognosis.

32 Blyme A, Asferg C, Nielsen OW et al. Increased hsCRP is associated with higher risk of aortic valve replacement in patients with aortic stenosis. Scand. Cardiovasc. J. 2(1), 138-145 (2016). 